\title{
Energy Consumption Analysis of a Novel Four- Speed Dual Motor Drivetrain for Electric Vehicles
}

\author{
T.Holdstock ${ }^{1}$, A.Sorniotti ${ }^{1}$, M.Everitt ${ }^{2}$, M.Fracchia ${ }^{2}$, S.Bologna ${ }^{3}$, S.Bertolotto ${ }^{3}$ \\ ${ }^{1}$ University of Surrey, ${ }^{2}$ Vocis Driveline Controls, ${ }^{3}$ Oerlikon Graziano - Automotive \\ Email: t.holdstock@surrey.ac.uk ${ }^{1}$
}

\begin{abstract}
The electric vehicle is becoming increasingly prevalent as a viable option to replace hydrocarbon fuelled vehicles, and as such the development of high efficiency fully electric drivetrains is a particularly relevant research topic. The drivetrain topology is one of the main focuses of research on fully electric drivetrains, because of the variety of available options. For example, the adoption of multiple-speed mechanical transmissions can improve both the performance and energy consumption when compared to a single-speed transmission. A four-speed, dual motor drivetrain design is presented in this article which works on the principle of two double-speed transmissions, each driven by a separate motor linked through a sole secondary shaft. This drivetrain architecture provides increased flexibility of the electric motor operating points, theoretically being beneficial to the overall efficiency of the system for any driving condition. This paper presents the design of the transmission, its governing equations and the method adopted to optimize the state selection map and electric motor torque distribution. A backward-facing energy consumption model is used to compare the results of the four-speed transmission with those of single- and double-speed transmissions for four case study vehicles.
\end{abstract}

\section{INTRODUCTION}

Multiple-speed mechanical transmission systems have been shown to increase the performance and efficiency of fully electric vehicles over single-speed transmissions when driven by a single electric motor [1-2], through increasing the range of the available wheel torques at the respective vehicle speed, and allowing the drivetrain to operate in higher efficiency regions. AMTs (Automated Manual Transmissions) are becoming a viable option for electric powertrains. Several AMTs have been developed specifically for electric drivetrains, for example [3]. However, a centrally located electric motor and a single-speed transmission are still the standard topological solution for electric vehicles presently being manufactured [4]. This is due to the favorable torque characteristic of an electric motor, being characterized by a constant torque region from zero to base speed, followed by a constant power region. Moreover, electric motors usually have a wide speed range, which can reach 10-15 $000 \mathrm{rpm}$.

The novel clutch-less four-speed dual motor transmission analyzed in this contribution, shown in Fig. 1, is unique in that it is powered by two electric motors, each driving a separate primary shaft, according to the conceptual schematic presented in [5]. Each primary shaft has two gears, the 'odd' gears being on one primary shaft, and the 'even' gears being on the opposing shaft, with the driven gear being selected via electro-mechanical dog clutch actuators driven by barrel cams. The four gears are directly coupled to the secondary

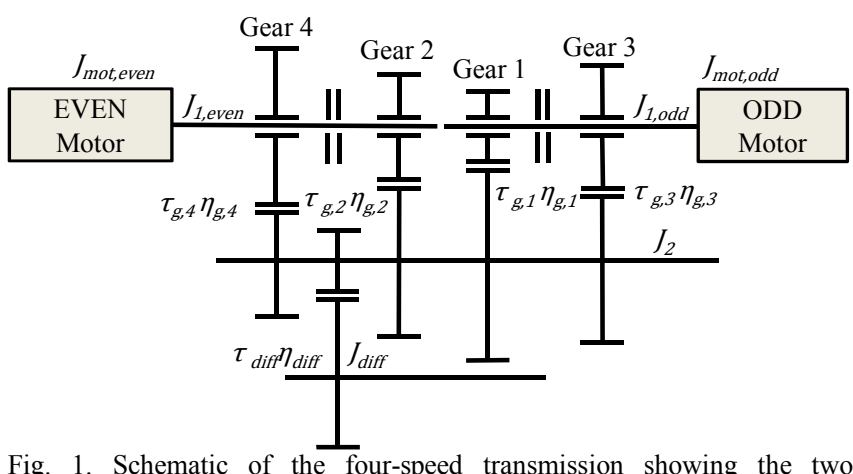

independent primary shafts and the lack of clutches for gearshift actuation. Nine possible states characterize the system operating conditions: 1) first and second gears engaged; 2) first and fourth gears engaged (not physically possible on the prototype under development); 3) second and third gears engaged; 4) third and fourth gears engaged; 5) only first gear engaged; 6) only second gear engaged; 7) only third gear engaged; 8) only fourth gear engaged; and 9) no engaged gear.

shaft which in turn is connected to the half-shafts via a final drive and a differential. The layout allows two low-power motors to be used as a replacement for a larger single electric motor, thus increasing the torque utilization factor of the motor drives, and potentially the operating efficiency along driving cycles.

The four-speed drivetrain provides a potential increase in dynamic performance over a single-speed drivetrain, because of the higher wheel torque at medium-low vehicle velocities, made available by high values of the first two gear ratios. The performance of the four-speed vehicle is comparable to the one of a double-speed. This is under the assumption, adopted for this research, that a motor with twice the power of the individual four-speed drivetrain motors is installed on the single- or double-speed case study vehicles. In conditions of high torque demand, a significant number of gearshifts are going to take place in the constant power region of the electric machine which can be detrimental to the drivability and longitudinal acceleration performance if a seamless shift is not achieved. In fact, if a gearshift is completed above the base speed of the electric motor for a constant accelerator pedal position, the vehicle acceleration is the same before and after the shift and thus any torque gap is well perceived. The clutch-less four-speed transmission permits the operation of seamless (in most operating conditions) gearshifts without the need for a synchronizer, by matching the primary shaft speeds electrically through controlling each motor during a gearshift.

The efficiency of an electric vehicle drivetrain varies across the operating speed/torque range as with an internal 
combustion engine, so the selection of the optimal states for each driving condition is especially important. Due to the architecture of this transmission, eight states are possible in driving conditions, increasing the possibility of the electric motors operating in a high efficiency area for a given wheel torque/vehicle speed combination. Fig. 2 shows fifteen areas where a different number of states, specified within the figure, are available to be used for an assigned driving condition. A higher number of available states indicates a higher probability of a high efficiency area of the motor/s being used. The maximum number of states is available at low vehicle speeds and wheel torques, which is the most frequent condition in typical driving cycles.

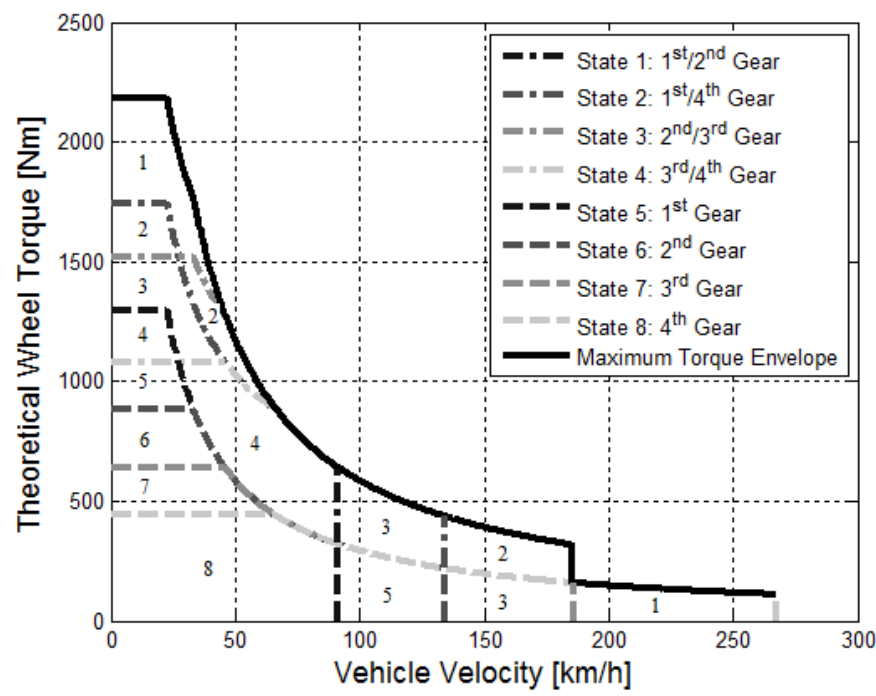

Fig. 2. Maximum wheel torque envelopes for each transmission state as a function of vehicle velocity. This plot illustrates the flexibility of the novel transmission system in the selection of the motor operating points.

[5] illustrates the basic theoretical advantage of this transmission design but provides little quantitative evidence of the energy consumption benefits. The work presented in this article, in collaboration with the industrial partners currently developing the physical prototype of the drivetrain concept, demonstrates the methods used to optimize the electric motor torque distribution and state selection, along with presenting the energy consumption results for one rear wheel drive, A, and three front wheel drive case study vehicles, B - D (see appendix for the vehicle data sets).

\section{TRANSMISSION AND VEHICLE MODELS}

A backward-facing model was developed for the energy consumption analysis of the single-, double- and four-speed case study vehicles. The same vehicle model was used for each case study vehicle, with only the drivetrain model changing to represent the respective transmission. The simulator accounts for rolling resistance, aerodynamic drag and inertial contributions along driving schedules. Moreover, a forward-facing model, including the first order drivetrain and motor air-gap torque dynamics, was implemented for the detailed simulation of gearshifts with the four-speed transmission. In this section the four-speed transmission governing equations will be presented along with a brief description of the vehicle models adopted.

Due to the four-speed transmission having two gears located on each primary shaft, the system can be described by three conditions, i) no engaged gear on either shaft; ii) an engaged gear on one shaft and no gear engaged on the opposing shaft; and iii) a gear engaged on each primary shaft. Consequently, the system has up to three degrees of freedom, as when no gear is selected on a primary shaft the motor can move freely whereas the secondary shaft and any selected gear is kinematically linked to the differential (not considering lash in the gears).

Each motor and primary shaft system can be represented by (1) when it is characterized by two disengaged gears and therefore as a separate degree of freedom.

$\begin{aligned} T_{\text {mot,odd/even }}-T_{\text {loss }, \text { odd/even }} & \\ & =\left(J_{\text {mot,odd/even }}+J_{1, \text { odd/even }}\right) \ddot{\vartheta}_{\text {mot,odd/even }}\end{aligned}$

The term $T_{\text {mot,even/odd }}$ represents the 'odd' and 'even' motor torques, $T_{\text {loss,even/odd }}$ is the 'odd' and 'even' motor losses due to windage and friction, $J_{1, \text { even } / o d d}$ is the moment of inertia of the 'even'/'odd' primary shafts, $J_{\text {mot,even } / o d d}$ is the moment of inertia of the 'even'/'odd' electric motors, $\ddot{\vartheta}_{\text {mot,odd/even. }}$ is the angular acceleration of the respective electric motor shaft.

The overall transmission system dynamics are described by the moment balance equation, (2), where the boolean variables flag $_{\text {sel,even/odd }}$ are used to indicate if a gear is selected or not selected on either the 'even' or 'odd' primary shaft.

$T_{\text {mot,even }} \tau_{g, \text { even,sel }} \eta_{g, \text { even,sel }} \tau_{\text {diff }} \eta_{\text {diff }}$ flag $g_{\text {sel,even }}$

$+T_{\text {mot,odd }} \tau_{g, o d d, s e l} \eta_{g, o d d, s e l} \tau_{\text {diff }} \eta_{\text {diff }}$ flag $g_{\text {sel,odd }}$

$-T_{\text {half-shaft,left }}-T_{\text {half-shaft,right }}$

$=\left[J_{\text {diff }}+J_{2} \tau_{\text {diff }}^{2} \eta_{\text {diff }}\right.$

$+\sum J_{g, \text { even,unsel }} \tau_{\text {g,even,unsel }}^{2} \eta_{g, \text { even,unsel }} \tau_{\text {diff }}^{2} \eta_{\text {diff }}$

$+\sum J_{\text {g,odd,unsel }} \tau_{\text {g,odd,unsel }}^{2} \eta_{g, \text { odd,unsel }} \tau_{\text {diff }}^{2} \eta_{\text {diff }}$

$+\left(\left(J_{\text {mot, }, \text { even }}+J_{1, \text { even }}+J_{\text {g,even,sel }}\right)\right.$ flag $\left._{\text {sel,even }}\right)$

$\tau_{g, e v e n, \text { sel }}^{2} \eta_{g, e v e n, \text { sel }} \tau_{\text {diff }}^{2} \eta_{\text {diff }}$

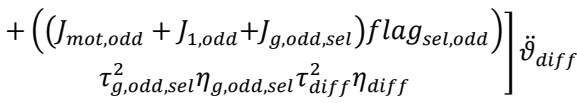

$\tau_{\text {g,even/odd,sel/unsel }}$ are the gear ratios of the selected or unselected gears on each shaft and $\eta_{\text {g,even/odd,sel/unsel }}$ are the respective equivalent efficiencies, $\tau_{\text {diff }}$ is the differential gear ratio, $\eta_{\text {diff }}$ is the differential efficiency, $T_{\text {half-shaft,left/right }}$ are the half-shaft torques, $J_{\text {diff }}$ is the moment of inertia of the differential, $J_{2}$ is the moment of inertia of the secondary shaft, $J_{g, \text { even/odd,sel/unsel }}$ are the moment of inertia of the selected or unselected gears on each 
shaft, and $\ddot{\vartheta}_{\text {diff }}$ is the angular acceleration of the differential. The four-speed drivetrain model includes experimentally attained efficiency maps for each motor drive and the mechanical transmission.

As an example of the operating principle of the novel fourspeed transmission, Fig. 3 illustrates the gearshift dynamics during a state $3\left(2^{\text {nd }} / 3^{\text {rd }}\right)$ to state $4\left(3^{\text {rd }} / 4^{\text {th }}\right)$ gearshift in power on, simulated through the forward facing model. Initially the torque is ramped down on the motor which will shift gears (Fig. 3i)), in this case the 'even' motor, as the 'even' primary shaft will shift from $2^{\text {nd }}$ to $4^{\text {th }}$, whilst the 'odd' primary shaft will remain in $3^{\text {rd }}$ gear throughout the shift. The 'odd' motor increases output torque to compensate for the 'even' motor torque reducing to zero. This is possible at low driver torque demands, however at high demands the compensating motor may become saturated, thus provoking a reduction in wheel torque resulting in a partial loss of vehicle acceleration. Once the 'even' motor torque has reduced to zero, the dog clutch disengages and the inertia phase starts to reduce the motor speed (Fig. 3iii)) to that of $4^{\text {th }}$ gear, through a feed-forward and Proportional Integral Derivative controller. Once the 'even' motor speed is equal to that of $4^{\text {th }}$ gear, the dog clutch re-engages and the torque is ramped up to the required level as designated by the EMS (Energy Management System). Fig. 3iv) shows that the vehicle acceleration remains approximately constant and illustrates the seamless shift capability of the system. From the motor torque profiles in Fig. 3i), it is evident that the gearshift process involves significant torque dynamics, especially during the inertia phase of the shift, when the electric synchronization is actuated, with the subsequent oscillations of the input power to the 'even' motor drive (evidenced by the circle of Fig. 3ii)). The inertia phase, especially when involving transition from a state with one active electric motor (states 5-8) to a state with two active electric motors (states 1-4), can imply a non-negligible effect on the overall energy balance along the driving cycle.

The backward-facing model does not simulate the gearshift dynamics in driving cycle simulations, for reasons of computational efficiency. As a consequence, the four-speed backward-facing model calculates the approximated energy $\Delta E_{\text {gearshift }}$, required to match the electric motor speeds during a gearshift, through the following equation:

$$
\begin{aligned}
& \Delta E_{\text {gearshift }}= \\
& =\eta_{\text {equiv,odd }}\left[\begin{array}{c}
f_{0, \text { odd }}\left(\frac{1}{2}\left(J_{\text {mot,odd }}+J_{1, \text { odd }}\right) \dot{\vartheta}_{\text {veh }}^{2} \tau_{\text {g,odd,next }}^{2} \tau_{\text {diff }}^{2}\right)- \\
f_{1, \text { odd }}\left(\frac{1}{2}\left(J_{\text {mot,odd }}+J_{1, \text { odd }}\right) \dot{\vartheta}_{\text {veh }}^{2} \tau_{\text {g,odd,prev }}^{2} \tau_{\text {diff }}^{2}\right)
\end{array}\right] \\
& +\eta_{\text {equiv,even }}\left[\begin{array}{c}
f_{0, \text { even }}\left(\frac{1}{2}\left(J_{\text {mot,even }}+J_{1, \text { even }}\right) \dot{\vartheta}_{\text {veh }}^{2} \tau_{\text {g,even,next }}^{2} \tau_{\text {diff }}^{2}\right)- \\
f_{1, \text { even }}\left(\frac{1}{2}\left(J_{\text {mot,even }}+J_{1, \text { even }}\right) \dot{\vartheta}_{\text {veh }}^{2} \tau_{\text {g,even,prev }}^{2} \tau_{\text {diff }}^{2}\right)
\end{array}\right]
\end{aligned}
$$

The equivalent efficiency of the electric motor is included through the term $\eta_{\text {equiv,even } / o d d}$ and the flags $f_{0 / 1 \text {,even/odd }}$ are either 0 or 1 depending on which state shift is taking place. In addition, $\dot{\vartheta}_{v e h}$ is the equivalent angular speed of the vehicle. The calculation is made for all the state transitions during a driving cycle and the sum added to the energy consumption of the two motor drives. The accuracy of the method used to estimate the additional energy required for a state shift was validated by comparing the results against the forward-facing model developed to analyze the gear shift dynamics of the four-speed transmission.

The backward-facing four-speed model uses look-up-tables

i)

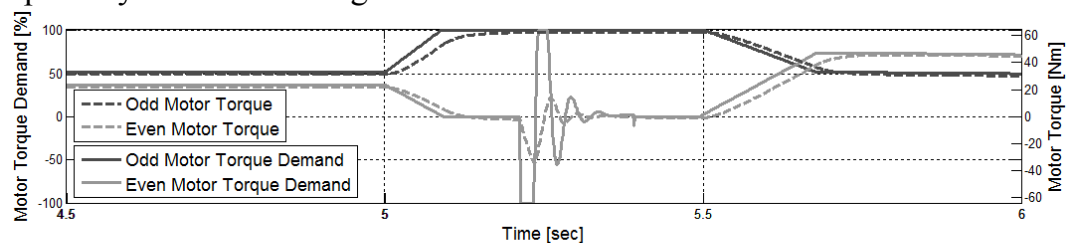

ii)

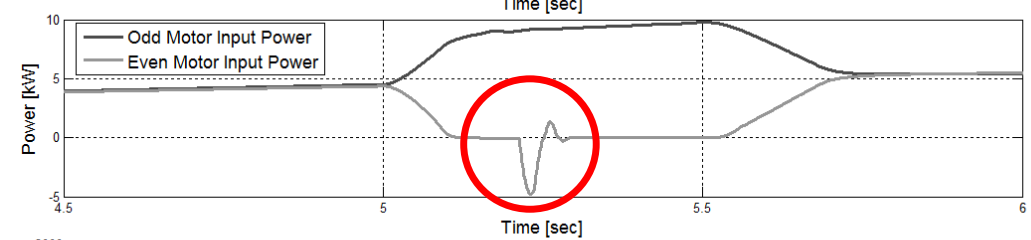

iii)
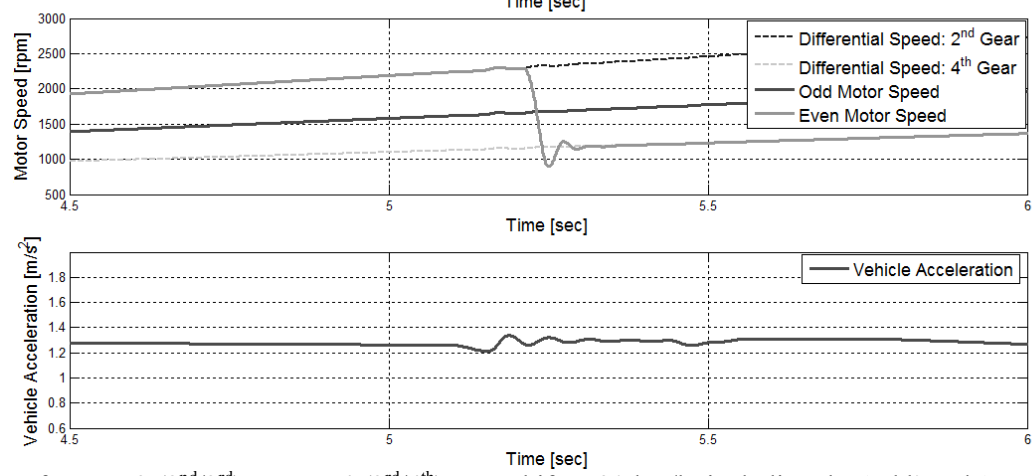

Fig. 3. The state shift dynamics of a state $3\left(2^{\text {nd }} / 3^{\text {rd }}\right)$ to state $4\left(3^{\text {rd }} / 4^{\text {th }}\right)$ state ${ }^{\text {Time }}$ shec] $20 \mathrm{~km} / \mathrm{h}$, including the 'odd' and 'even' motor torque demands and actual torques (i), electric motor input powers (ii) and speeds (iii) for Case C. The acceleration profile (iv) of the vehicle during the shift is also included. The terms 'Differential speed: $2^{\text {nd }} / 4^{\text {th }}$ Gear' in the legend shown in iii) refer to the differential speed at the motor in the respective gear. 
to select the state and the 'odd' motor torque (the latter only when the concurrent operation of the two motors is required), using the vehicle speed and required wheel torque as inputs. The 'even' motor torque is then calculated within the model, starting from the wheel torque and the 'odd' motor torque. The EMS also defines the regenerative torque limits required by the brake regulations [6].

\section{THE OPTIMIZATION PROCEDURES}

Each of the case study vehicles underwent a gear ratio optimization for the single-, double- and four-speed systems. In addition, the double-speed transmission requires the optimization of the state selection (gearshift map), whilst the four-speed transmission requires both the state selection and the torque distribution between the two motors to be tuned for each driving condition.

The selection of the optimal transmission parameters is based on cost functions. Similarly to what described in [7], two separate cost functions were used, namely the DPCF (Dynamic Performance Cost Function) and the ECCF (Energy Consumption Cost Function). The DPCF is based on acceleration tests, for the derivation of relevant performance indicators such as vehicle acceleration times and top speeds at different road gradients. The DPCF also includes static calculations of the maximum achievable road gradient at different vehicle velocities. The individual results are combined through weighting factors to generate the values of the DPCF for different transmission parameters. The ECCF considers the energy consumption along a weighted selection of driving cycles, such as the NEDC (New European Driving Cycle) and the FTP-75 (Federal Testing Procedure). In the case of the double- and four-speed vehicles, the ECCF and DPCF adimensionalize each value of the performance indicators with a 'base' result obtained through the optimized single-speed vehicle. As a consequence, a cost function value lower than one indicates an improvement over the singlespeed vehicle. Fig. 4 shows an example of a surface plot of the ECCF for a wide range of the adimensionalized (through the constant values, $\tau_{\text {ISTD }}$ and $\tau_{\text {2STD }}$ ) first and second gear ratios ( $\tau_{1}$ and $\tau_{2}$ respectively), and two gearshift maps ('map STD' and 'map 1'), obtained for a double-speed vehicle.

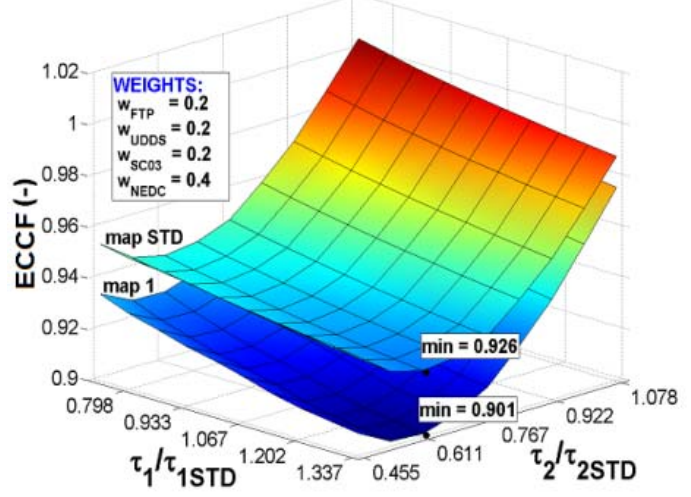

Fig. 4. An example of the results obtained for the ECCF of a double-speed case study vehicle. The gear ratios tested are represented as functions of the standard (initial) gear ratio selected, $\tau_{1 S T D}$ and $\tau_{2 S T D}$
For the four-speed vehicles, initially the forward-facing model is used as a computationally efficient preliminary test to verify that the longitudinal acceleration performance is

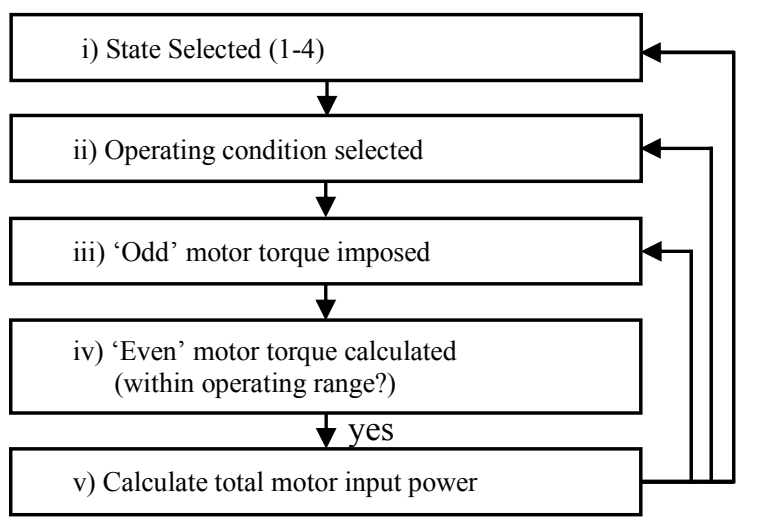

Fig. 5. Flow chart illustrating the off-line steps carried out to optimize the motor torque distribution for the dual gear states (1-4) of the four-speed transmission.

within the assigned constraints for the DPCF. Then, for each set of gear ratios satisfying the performance constraints, the optimal state and torque distribution between the two motors are selected for every achievable wheel torque and vehicle speed combination. These values are used for creating the look-up-tables required by the EMS implemented in the backward-facing model used for the simulation of the driving cycles required for the ECCF. To this purpose, an iterative

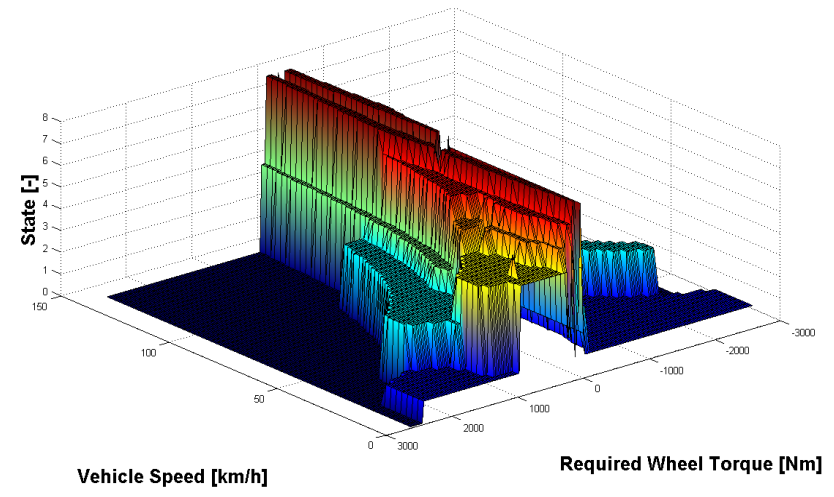

Fig. 6. Example of a state look-up-table, giving the optimal state for each driving condition for case $\mathrm{C}$.

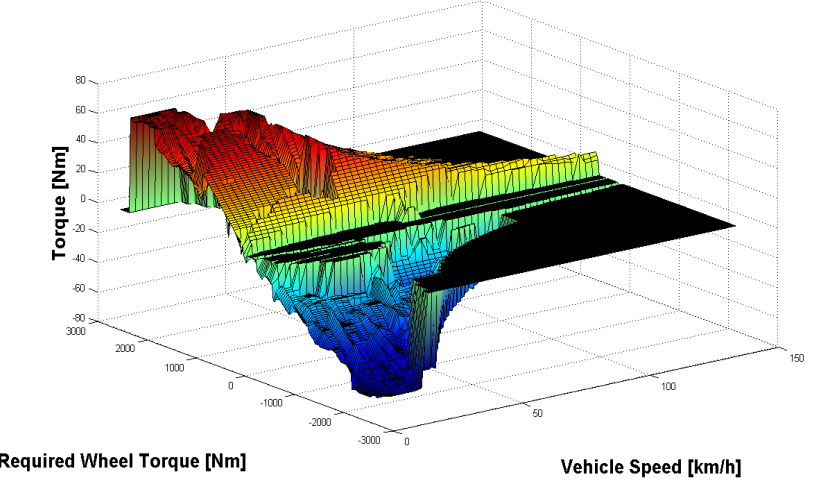

Fig. 7. Example of an 'odd' motor torque look-up-table, giving the optimal torque demand for each driving condition for case $\mathrm{C}$. 
algorithm is adopted to calculate the input power to the electric drivetrain for each of the eight relevant transmission states and wheel torque/vehicle speed conditions. In addition to the gear ratios and the steady-state efficiency contributions of the different subsystems, the off-line procedure accounts for the inertial torques of the drivetrain components. For the dual gear states (Fig. 5), initially the air gap torque is imposed (Fig. 5iii)) on the 'odd' motor and the 'even' motor torque (Fig 5iv)) is calculated to achieve the wheel torque for the set operating condition (imposed in step ii) of Fig. 5). If the 'even' motor torque falls outside the operating range of the motor, the result is not considered. The procedure is carried out for the whole range of available 'odd' motor torques. For the states where a single gear is selected, a backwards calculation of the required input drivetrain power starting from the wheel torque/speed is used. Following the completion of the procedure for each of the eight states, the most energy efficient state (i.e., the state requiring the smallest input power to the electric drivetrain) is selected for each wheel torque and vehicle speed combination. This results in maps such as those of Figs. 6 and 7, showing the optimal state number and 'odd' motor torque for every achievable operating condition. The state selection look-up- tables for the four-speed vehicles incite a single gear to be selected in traction at low torques to reduce the inertia the system is required to overcome, thus decreasing the energy consumption. This is illustrated in the state selection map shown in Fig. 6. During braking, a state with two gears tends to be engaged to increase the inertia of the system and the regenerated energy. This advantage is only apparent as it is compensated by the energy required for the transition from a state with only one operating motor to a state with two operating motors, estimated through (3). The state selection maps derived through the optimization procedure have to be smoothed to guarantee acceptable drivability. In addition, the actual state selection strategy must be characterized by a hysteresis in the transition between the states, similarly to the standard practice in the design of the gearshift maps for an automated transmission [2]. In the final step of the optimization procedure of the four-speed system, the backward-facing model calculates the energy consumption along the specified driving cycles, which allows the computation of the ECCF value for each set of gear ratios.

\section{RESULTS}

Each of the case study vehicles were simulated with the

Table 1. Energy consumption at the electric motor drive for each of the four case study vehicles, A - D, for the NEDC and FTP-75 driving cycles. The percentage difference is found through the formula (here the double-speed to single-speed comparison is used as an example):

Percentage Difference $=\left(\left(\right.\right.$ Energy $y_{\text {double-speed }}$-Energy single-speed $) /$ Energy $\left._{\text {single-speed }}\right) 100$

\begin{tabular}{|c|c|c|c|c|c|c|}
\hline & $\begin{array}{c}\text { Single-Speed } \\
{[\mathrm{kWh}]}\end{array}$ & $\begin{array}{c}\text { Double-Speed } \\
{[\mathrm{kWh}]}\end{array}$ & $\begin{array}{l}\text { Double-Speed/ } \\
\text { Single-Speed } \\
\text { Percentage } \\
\text { Difference [\%] }\end{array}$ & $\begin{array}{c}\text { Four-Speed } \\
{[\mathrm{kWh}]}\end{array}$ & $\begin{array}{l}\text { Four-Speed/ } \\
\text { Single-Speed } \\
\text { Percentage } \\
\text { Difference [\%] }\end{array}$ & $\begin{array}{l}\text { Four-Speed/ } \\
\text { Double-Speed } \\
\text { Percentage } \\
\text { Difference [\%] }\end{array}$ \\
\hline \multicolumn{7}{|c|}{ Case A } \\
\hline NEDC - Total energy & 1.65 & 1.63 & -1.2 & 1.55 & -5.6 & -4.4 \\
\hline NEDC - Energy in traction & 2.09 & 2.08 & -0.6 & 2.03 & -2.7 & -22.1 \\
\hline NEDC - Energy in regeneration & -0.44 & -0.45 & 1.4 & -0.48 & 7.8 & 6.4 \\
\hline FTP-75 - Total energy & 2.59 & 2.57 & -0.6 & 2.49 & -3.8 & -3.2 \\
\hline FTP-75 - Energy in traction & 3.47 & 3.47 & 0.1 & 3.45 & -0.7 & -0.8 \\
\hline FTP-75 - Energy in regeneration & -0.88 & -0.90 & 2.1 & -0.96 & 8.4 & 6.1 \\
\hline \multicolumn{7}{|c|}{ Case B } \\
\hline NEDC - Total energy & 1.08 & 1.02 & -5.5 & 0.98 & -9.0 & -3.8 \\
\hline NEDC - Energy in traction & 1.19 & 1.13 & -5.1 & 1.15 & -3.9 & 1.3 \\
\hline NEDC - Energy in regeneration & -0.11 & -0.11 & -1.8 & -0.16 & 47.4 & 50.2 \\
\hline FTP-75 - Total energy & 1.53 & 1.45 & -5.1 & 1.44 & -5.6 & -0.5 \\
\hline FTP-75 - Energy in traction & 1.80 & 1.70 & -5.2 & 1.76 & -2.0 & 3.4 \\
\hline FTP-75 - Energy in regeneration & -0.27 & -0.25 & -5.7 & -0.32 & 18.3 & 25.5 \\
\hline \multicolumn{7}{|c|}{ Case $\mathrm{C}$} \\
\hline NEDC - Total energy & 1.89 & 1.53 & -18.9 & 1.40 & -26.1 & -8.8 \\
\hline NEDC - Energy in traction & 2.13 & 1.79 & -16.2 & 1.66 & -21.9 & -6.9 \\
\hline NEDC - Energy in regeneration & -0.24 & -0.25 & 5.9 & -0.26 & 11.3 & 5.1 \\
\hline FTP-75 - Total energy & 2.71 & 2.34 & -13.8 & 2.04 & -24.7 & -12.7 \\
\hline FTP-75 - Energy in traction & 3.26 & 2.90 & -11.0 & 2.68 & -17.8 & -7.7 \\
\hline FTP-75 - Energy in regeneration & -0.54 & -0.56 & 2.9 & -0.64 & 16.6 & 13.3 \\
\hline \multicolumn{7}{|c|}{ Case D } \\
\hline NEDC - Total energy & 1.63 & 1.46 & -10.7 & 1.00 & -38.6 & -31.2 \\
\hline NEDC - Energy in traction & 1.74 & 1.57 & -10.0 & 1.15 & -34.1 & -26.8 \\
\hline NEDC - Energy in regeneration & -0.11 & -0.11 & 1.0 & -0.15 & 30.6 & 29.3 \\
\hline FTP-75 - Total energy & 2.23 & 2.09 & -6.5 & 1.43 & -35.8 & -31.4 \\
\hline FTP-75 - Energy in traction & 2.52 & 2.39 & -5.3 & 1.77 & -29.9 & -26.0 \\
\hline FTP-75 - Energy in regeneration & -0.29 & -0.30 & 3.7 & -0.33 & 16.0 & 11.9 \\
\hline
\end{tabular}


optimized single-speed, double-speed and novel four-speed transmissions along the NEDC and FTP-75 driving cycles. The results are given in Table 1. The simulations show a reduction in energy consumption between the single- and double-speed vehicles consistent with the research in [2], with an improvement up to $19 \%$ for the NEDC for case C, although the reduction is less than $1 \%$ for the FTP-75 for case A. The four-speed vehicles allow a consistent improvement over the single-speed equivalent for each case study and driving cycle, ranging from $6 \%$ (case A) to $39 \%$ (case D) for the NEDC and from 4\% (case A) to $36 \%$ (case D) for the FTP-75. The improvement of the four-speed drivetrain over the double-speed equivalent ranges from $4 \%$ (case B) to $31 \%$ (case D) for the NEDC and from $1 \%$ (case B) to $31 \%$ (case D) for the FTP-75. The energy consumption reduction for the four-speed vehicle is due to both a reduction in the energy consumed in traction and an increase in the energy regenerated during braking along each driving cycle.

Fig. 8 plots the electric motor input power over the NEDC for the double-speed case $\mathrm{C}$ vehicle and the sum of the two motors for the four-speed equivalent. The double-speed vehicle uses more power throughout the driving cycle, especially in condition of constant velocity. The significant power difference at constant speed is due to the fact that the motor torque demand is low, where the motor efficiency map is characterized by a significant gradient. The four-speed system can rely on a single motor drive at low torque demands, with a doubled utilization of the torque capability of the motor when compared to the double-speed, and thus works in a much more efficient point.

In addition, the vehicle performance of each four-speed case study vehicle was found to be comparable to the doublespeed variants with similar acceleration times and top-speeds. However, a significant increase in performance was found when comparing the four-speed and double-speed variant results against the single-speed.

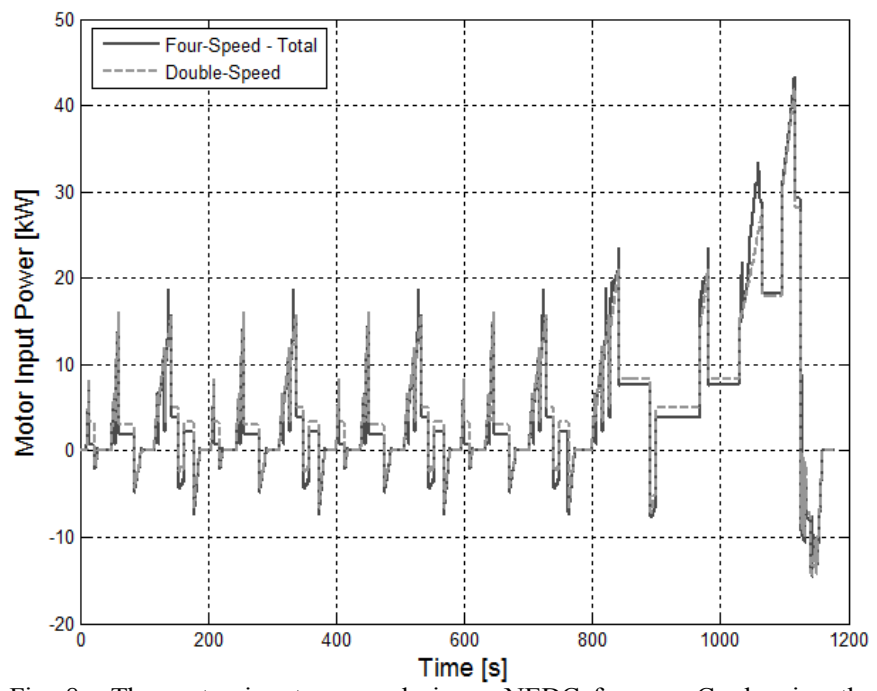

Fig. 8. The motor input power during a NEDC for case $\mathrm{C}$, showing the double-speed motor power against the sum of the 'odd' and 'even' motor powers for the four-speed drivetrain.

\section{CONCLUSION}

This paper has dealt with the energy consumption analysis of a novel four-speed clutch-less transmission system driven by two independent electric motors. A description of the fourspeed transmission has been included along with the governing equations and the method used to optimize the state selection and motor torque distribution for each driving condition. An explanation of the gearshift dynamics has been included together with a method adopted to account for the shift energy in a backward-facing model. Four case study vehicles were analyzed in the research, each of them characterized by electric motors with different powers, peak torque and maximum speeds. The four-speed vehicles were proven to achieve an energy consumption reduction over standard driving cycles against single and double-speed variants of each case study vehicle.

\section{REFERENCES}

[1] Ren, Q., Crolla, D.A., Morris, A., "Effect of transmission design on electric vehicle (EV) performance", IEEE Vehicle Power and Propulsion Conference, September 2009.

[2] Sorniotti, A., Subramanyan, S., Turner, A., Cavallino, C. et al., "Selection of the optimal gearbox layout for an electric vehicle", SAE Int. J. Engines 4(1): 1267-1280, 2011.

[3] Sorniotti, A., Holdstock, T., Loro Pilone, G. et al., "Analysis and simulation of the gearshift methodology for a novel two-speed transmission system for electric powertrains with a central motor", Proceedings of the Institution of Mechanical Engineers, Part D: Journal of Automobile Engineering, Vol. 226, Issue 7, pp 915 - 929, July 2012.

[4] http://www.renault.co.uk/cars/model/twizy/product.aspx, last consulted on $28^{\text {th }}$ June 2012.

[5] Rinderknecht, S., Meier, T., Fietzek, R., "Electric two-drive-transmission for E-vehicles", VDI-Berichte, No.2130, 2011.

[6] http://www.unece.org/trans/main/wp29/wp29regs1-20.html, last consulted on $6^{\text {th }}$ July 2012.

[7] Sorniotti, A., Boscolo, M., Turner, A., Cavallino, C., "Optimization of a multi-speed electric axle as a function of the electric motor properties", IEEE Vehicle Power and Propulsion Conference, September 2010.

\section{APPENDIX}

Table A.1. Case study vehicle parameters

\begin{tabular}{|l|c|c|c|c|}
\hline & Case A & Case B & Case C & Case D \\
\hline Vehicle mass [kg] & 2109 & 650 & 1300 & 750 \\
\hline Wheelbase [m] & 2.8 & 2 & 2.57 & 1.87 \\
\hline Wheel radius [m] & 0.327 & 0.31 & 0.31 & 0.283 \\
\hline Coefficient of drag [-] & 0.28 & 0.32 & 0.30 & 0.38 \\
\hline Frontal area [m²] & 2.6 & 2.2 & 2.5 & 1.8 \\
\hline $\begin{array}{l}\text { Max. motor power [kW] } \\
\text { (single-/double-speed) }\end{array}$ & 333 & 50 & 50 & 50 \\
\hline $\begin{array}{l}\text { Max. motor torque [Nm] } \\
\text { (single-/double-speed) }\end{array}$ & 800 & 160 & 128 & 128 \\
\hline $\begin{array}{l}\text { Max. motor power [kW] } \\
\text { (four-speed) }\end{array}$ & 168 & 25 & 25 & 25 \\
\hline $\begin{array}{l}\text { Max. motor torque [Nm] } \\
\text { four-speed) }\end{array}$ & 400 & 80 & 64 & 64 \\
\hline Max. motor speed [rpm] & 5000 & 14000 & 15000 & 15000 \\
\hline \multicolumn{4}{|l|}{ Optimized Four-Speed Gear Ratios (excluding $\left.\tau_{\text {diff }}\right)$} \\
\hline First gear ratio [-] & 3.188 & 4.5 & 4.5 & 3.8 \\
\hline Second gear ratio [-] & 2.227 & 4 & 4 & 2.5 \\
\hline Third gear ratio [-] & 1.025 & 2.25 & 2 & 1.9 \\
\hline Fourth gear ratio [-] & 1 & 1.25 & 1.1 & 1.1 \\
\hline
\end{tabular}

